\title{
Non-histologic factors discriminating proliferative lupus nephritis from membranous lupus nephritis
}

Oh Chan Kwon', Jung Hwan Park', Hyeong-Cheon Park², Seung Min Jung ${ }^{1}$, Sang-Won Lee', Jason Jungsik Song ${ }^{1}$, Yong-Beom Park ${ }^{1}$ and Min-Chan Park ${ }^{1 *}$ (i)

\begin{abstract}
Background: To investigate non-histologic factors that can discriminate proliferative lupus nephritis (LN) from membranous $L N$ in patients with systemic lupus erythematosus with renal manifestations.

Methods: Patients with biopsy-proven proliferative LN (class III \pm V and class IV $\pm \mathrm{V}$ ) and membranous LN (class V) were included. Non-histologic factors were compared between the two groups. A logistic regression analysis was performed to identify the factors associated with proliferative LN. To assess the accuracy of these factors in discriminating between proliferative $L N$ and membranous $L N$, we performed a receiver-operating characteristic analysis.

Results: Of the total 168 patients with biopsy-proven LN, 150 patients (89.3\%) had proliferative LN, and 18 patients (10.7\%) had membranous LN. In the multivariable logistic regression analysis, positive anti-double-stranded DNA (antidsDNA) antibody (adjusted $\mathrm{OR}=11.200,95 \% \mathrm{Cl}=2.202-56.957, p=0.004$ ) was associated with proliferative $L N$, while positive anti-U1RNP antibody (adjusted $\mathrm{OR}=0.176,95 \% \mathrm{Cl}=0.040-0.769, p=0.021$ ) and higher glomerular filtration rate (GFR) (adjusted $\mathrm{OR}=0.973,95 \% \mathrm{Cl}=0.951-0.994, p=0.013$ ) were inversely associated with proliferative $\mathrm{LN}$. Among these covariates, the anti-dsDNA antibody (area under the curve $=0.806,95 \% \mathrm{Cl}=0.695-0.916$ ) had the highest accuracy in discriminating between proliferative $L N$ and membranous $L N$.

Conclusion: The positivity of anti-dsDNA antibody was associated with proliferative LN, while the positivity of anti-U1RNP antibody and GFR were inversely associated with proliferative LN. The anti-dsDNA antibody had a good accuracy in discriminating proliferative LN from membranous LN.
\end{abstract}

Keywords: Systemic lupus erythematosus, Lupus nephritis, Proliferative, Membranous

\section{Background}

Lupus nephritis (LN) is one of the common manifestations of systemic lupus erythematosus (SLE) that causes significant morbidity and mortality [1]. According to the International Society of Nephrology/Renal Pathology Society 2003 classification, LN is classified into six classes according

\footnotetext{
* Correspondence: mcpark@yuhs.ac

'Division of Rheumatology, Department of Internal Medicine, Yonsei University College of Medicine, Seoul, South Korea

Full list of author information is available at the end of the article
}

to the glomerular pathology [2]. Among these classes, class III, class IV, and class V have the potential to cause longterm renal damage [3]. Class III and class IV (proliferative $\mathrm{LN}$ ) are highly inflammatory with immune complex deposition in the subendothelial space, whereas class $\mathrm{V}$ (membranous LN) is less inflammatory with immune complex deposition in the subepithelial space [2]. Proliferative LN is usually treated with potent immunosuppressive agents, whereas membranous LN may be managed conservatively with antiproteinuric agents if patients have subnephrotic proteinuria or with immunosuppressive agents if patients 
have nephrotic-range proteinuria $[4,5]$. The risk of progression to end-stage renal disease (ESRD) differs between proliferative $\mathrm{LN}$ and membranous $\mathrm{LN}$, with proliferative $\mathrm{LN}$ having a worse prognosis (risk of ESRD, 10-20\% for proliferative LN vs $<10 \%$ for membranous LN) $[4,6]$. Considering the differences in the treatment strategy and renal prognosis between proliferative LN and membranous LN, it is important to distinguish one from the other.

The confirmative modality for diagnosing LN and distinguishing proliferative $\mathrm{LN}$ from membranous $\mathrm{LN}$ is renal biopsy [5]. The American College of Rheumatology (ACR) recommends renal biopsies in patients with SLE who have increasing serum creatinine levels of an unknown cause, proteinuria at protein levels of $\geq 1 \mathrm{~g}$ per day (either in a 24-h urine specimen or on a spot protein/creatinine ratio [PCR]), or a combination of the following: proteinuria at protein levels of $\geq 0.5 \mathrm{~g}$ per day plus $\geq 5$ red blood cells (RBCs) per high power field (HPF) or proteinuria at protein levels of $\geq 0.5 \mathrm{~g}$ per day plus cellular casts [7]. Although it is apparently important to perform renal biopsies to confirm the diagnosis of LN and to guide appropriate therapeutic decisionmaking based on the classification of LN, there are some circumstances where renal biopsies are difficult to perform, such as in patients under mechanical ventilation who have difficulty in assuming the prone position, patients with uncorrectable bleeding diathesis, and patients with small kidney sizes. Given that renal biopsies may not always be available, it is clinically meaningful to identify non-histologic factors that can discriminate proliferative LN from membranous LN.

To date, data regarding clinical factors predictive of proliferative LN are limited. In this study, we aimed to identify non-histologic factors predictive of proliferative LN.

\section{Materials and methods Patients}

Data from two independent LN cohorts from two tertiary referral hospitals in Seoul, Korea, were retrospectively reviewed. Both cohorts consisted of patients diagnosed with LN via renal biopsy between July 2006 and December 2018. All patients met the 1997 ACR classification criteria for SLE [8]. Renal biopsies were performed in accordance with the indications recommended by the ACR [7]. The patients were categorized into the proliferative LN (class III, class IV, class III $+\mathrm{V}$ and class IV $+\mathrm{V}$ ) and membranous LN (class V) groups based on their renal biopsy reports. Given that the therapeutic strategy is similar between pure proliferative LN (class III and class IV) and mixed proliferative LN (class III + V and class IV $+V$ ), and that the therapeutic strategy in both is different from that in membranous LN [7, 9], pure proliferative LN (class III and class IV) and mixed proliferative LN (class III $+\mathrm{V}$ and class IV $+\mathrm{V}$ ) were both categorized as proliferative LN in the primary analysis. As the purpose of this study was to identify the factors that discriminate proliferative LN from membranous LN, patients with class I, class II, and class VI were excluded. This study was approved by the Institutional Review Board (IRB) of Gangnam Severance Hospital (IRB No: 3-2019-0072). Owing to the retrospective nature of this study, the requirement for informed consent was waived.

\section{Covariates}

Data on the following covariates at the time of renal biopsy were collected: age, sex, presence of hypertension and diabetes mellitus, manifestations of SLE other than those of $\mathrm{LN}$, positivity of antibodies (Abs) to extractable nuclear antigens, anti-double-stranded DNA (anti-dsDNA) Ab, lupus anticoagulant, anti- $\beta_{2}$ glycoprotein $\mathrm{Ab}$ and anti-cardiolipin $\mathrm{Ab}, \mathrm{C} 3$ and $\mathrm{C} 4$ levels, serum albumin and creatinine levels, glomerular filtration rate (GFR), urine PCR, urinalysis results, and SLE Disease Activity Index 2000 (SLEDAI-2 K) [10]. Autoantibodies were measured using an automated fluoroimmunoassay analyzer (EliA; Phadia, Uppsala, Sweden). Lupus anticoagulants were assessed using the IL Test TM LAC Screen/Confirm Kit (Instrumentation Laboratory Co., Bedford, MA, USA).

\section{Statistical analysis}

The patients' characteristics were summarized using descriptive statistics. To compare the characteristics between the proliferative LN group and membranous LN group, Student's $t$ test or Mann-Whitney test was used for continuous variables and Fisher's exact test or chisquare test (when appropriate) for categorical variables. Multivariable logistic regression models were constructed to identify the covariates associated with proliferative LN. Covariates with a $p$ value of $<0.05$ in the univariable logistic regression analysis were incorporated to the multivariable models. In the multivariable analysis, the variable inflation factor was tested to exclude multicollinearity among covariates. The Hosmer-Lemeshow test was used to assess the goodness of fit for the logistic regression models. Antibodies and complements ( $\mathrm{C} 3$ and $\mathrm{C} 4)$ were analyzed as binary variables (positive/negative for antibodies, and low/not low for complements) in univariable analysis and multivariable analysis (model 1) and were analyzed as continuous variables in multivariable analysis (model 2). We used a receiver-operating characteristic (ROC) analysis to assess the ability of the covariates identified in the multivariable models in discriminating proliferative LN from membranous LN. ROC curves were generated, and the associated area under the curve (AUC) for each covariate was determined. The statistical significance level was set at a $p$ value of $<0.05$. All analyses were 
conducted using the SPSS software (version 25.0; IBM Corporation, Armonk, NY, USA).

\section{Sensitivity analysis}

To test the robustness of our results, we performed several sensitivity analyses. First, we used a more restrictive definition of proliferative LN. Instead of including both pure proliferative LN (class III and class IV) and mixed proliferative LN (class III $+\mathrm{V}$ and class IV $+\mathrm{V}$ ) in the proliferative LN group, we included only the pure proliferative LN (class III and class IV) in the proliferative LN group and performed multivariable logistic regression analysis and ROC analysis. Second, we compared mixed proliferative LN (class III $+\mathrm{V}$ and class IV + V) with membranous LN (class V). Third, as patients with classes I, II, and VI would also have to undergo a renal biopsy for diagnostic purposes, we included the patients with classes I, II, and VI and compared pure proliferative LN (class III and class IV) with non-proliferative LN (class I, class II, class V, and class VI).

\section{Results}

\section{Patient characteristics}

A total of 176 patients with biopsy-proven LN were included. The patients were predominantly women (90.9\%), with a mean age of $36.7 \pm 15.0$ years. Among the patients, 150 patients $(85.2 \%)$ had proliferative LN, and 18 patients (10.2\%) had membranous LN. Of the 150 patients with proliferative LN, $122(81.3 \%)$ patients had pure proliferative LN (class III, 38 patients; class IV, 84 patients) and 28 (18.7\%) patients had mixed proliferative LN (class III + V, 17 patients; class IV + V, 11 patients) (Table 1). Three (1.7\%) patients with class I, four $(2.3 \%)$ patients with class II, and one (0.6\%) patient with class VI were excluded for primary analysis.

Table 1 Histologic characteristics of the 176 patients

\begin{tabular}{ll}
\hline & $N=176$ \\
\hline ISN/RPS class & \\
I, $n(\%)$ & $3(1.7 \%)$ \\
II, $n(\%)$ & $4(2.3 \%)$ \\
III, $n(\%)$ & $38(21.6 \%)$ \\
IV,$n(\%)$ & $84(47.7 \%)$ \\
III + V, n (\%) & $17(9.7 \%)$ \\
IV + V, n (\%) & $11(6.3 \%)$ \\
$V, n(\%)$ & $18(10.2 \%)$ \\
VI, $n(\%)$ & $1(0.6 \%)$ \\
Activity index, median (IQR) & $7.0(3.0-11.0)$ \\
Chronicity index median (IQR) & $1.0(0.5-2.5)$ \\
\hline
\end{tabular}

ISN/RPS International Society of Nephrology/Renal Pathology Society, IQR interquartile range
The comparison of the characteristics between the two groups is shown in Table 2. Age $(p=0.269)$, sex distribution $(p=0.649)$, and the proportion of patients with hypertension $(p>0.999)$ and diabetes mellitus $(p=0.599)$ did not differ between the two groups. The proportion of patients with mucocutaneous manifestations $(p>0.999)$, musculoskeletal manifestations $(p=0.408)$, neuropsychiatric manifestations $(p=0.149)$, and serositis $(p=0.475)$ was similar in the two groups; conversely, hematologic manifestations were more common in the patients with proliferative $\mathrm{LN}(44.7 \%$ vs $16.7 \%, p=0.023)$. In the comparison of serologic covariates, no significant differences were observed in the positivity for anti-Sm $\mathrm{Ab}(p=0.384)$, anti-Ro $\mathrm{Ab}(p=0.885)$, anti-La $\mathrm{Ab}(p=0.258)$, lupus anticoagulant $(p=0.768)$, anti- $\beta_{2}$ glycoprotein $\mathrm{Ab}(p=0.077)$, and anti-cardiolipin $\mathrm{Ab}(p=0.566)$ and albumin levels $(p=0.800)$. The patients with proliferative $\mathrm{LN}$ were less commonly positive for anti-U1RNP Ab (48.7\% vs $77.8 \%$, $p=0.020)$ and more commonly positive for anti-dsDNA Ab $(88.0 \%$ vs $50.0 \%, p<0.001)$, and more commonly had low C3 $(94.0 \%$ vs $61.1 \%, p<0.001)$ and low C4 $(79.3 \%$ vs $50.0 \%, p=0.015)$, higher creatinine level $(1.13 \pm 0.79 \mathrm{mg} /$ $\mathrm{dL}$ vs $0.70 \pm 0.26 \mathrm{mg} / \mathrm{dL}, \mathrm{p}<0.001)$, and lower GFR $\left(83.2 \pm 37.0 \mathrm{~mL} / \mathrm{min} / 1.73 \mathrm{~m}^{2}\right.$ vs $105.1 \pm 23.8 \mathrm{~mL} / \mathrm{min} / 1.73$ $\left.\mathrm{m}^{2}, p=0.002\right)$. Regarding the urine laboratory data, the urine PCR $(p=0.778)$ and proportion of patients with pyuria $(p=0.053)$ and urine casts $(p=0.202)$ did not differ between the two groups. The proportion of patients with urine $\mathrm{RBC}$ of $\geq 5 / \mathrm{HPF}$ was higher in the proliferative $\mathrm{LN}$ group $(72.7 \%$ vs $38.9 \%, p=0.003)$. The SLEDAI- $2 \mathrm{~K}$ was also higher in the proliferative LN group $(17.1 \pm 5.8$ vs $12.2 \pm 5.8, p=0.001)$.

\section{Covariates associated with proliferative $\mathrm{LN}$}

In the univariable logistic regression analysis, the presence of hematologic manifestations (unadjusted odds ratio $[\mathrm{OR}]=4.036,95 \%$ confidence interval $[\mathrm{CI}]=1.121-$ 14.527, $p=0.033$ ), positive anti-dsDNA Ab (unadjusted $\mathrm{OR}=7.333,95 \% \mathrm{CI}=2.574-20.893, p<0.001)$, low C3 (unadjusted OR $=9.970,95 \%$ CI 3.117-31.891, $p<$ 0.001 ), low C4 (unadjusted OR 3.839 , 95\% CI $1.405-$ 10.486, $p=0.009$ ), creatinine levels (unadjusted $\mathrm{OR}=$ $10.645,95 \% \mathrm{CI}=1.350-83.953, p=0.025)$, presence of urine $\mathrm{RBC}$ of $\geq 5 / \mathrm{HPF}$ (unadjusted $\mathrm{OR}=4.718,95 \%$ $\mathrm{CI}=1.516-11.509, p=0.006)$, and higher SLEDAI-2 K (unadjusted $\mathrm{OR}=1.173,95 \% \mathrm{CI}=1.063-1.294, p=$ $0.001)$ were associated with proliferative $L N$. The positivity of anti-U1RNP Ab (unadjusted OR $=0.271,95 \%$ $\mathrm{CI}=0.085-0.861, \quad p=0.027$ ) and GFR (unadjusted $\mathrm{OR}=0.980,95 \% \mathrm{CI}=0.964-0.997, p=0.021)$ were inversely associated with proliferative $\mathrm{LN}$. These covariates were included in the multivariable models, except for the creatinine level, because of the multicollinearity with the GFR. 
Table 2 Comparison of the clinical characteristics between the patients with proliferative LN and membranous LN

\begin{tabular}{|c|c|c|c|}
\hline & Proliferative LN $(N=150)$ & Membranous LN $(N=18)$ & $p$ \\
\hline Age, mean $( \pm S D)$, years & $35.8( \pm 14.3)$ & $39.8( \pm 15.4)$ & 0.269 \\
\hline Female sex, $n(\%)$ & $138(92.0 \%)$ & $16(88.9 \%)$ & 0.649 \\
\hline Hypertension, $n$ (\%) & $34(22.7 \%)$ & $4(22.2 \%)$ & $>0.999$ \\
\hline Diabetes mellitus, $n$ (\%) & $9(6.0 \%)$ & $0(0.0 \%)$ & 0.599 \\
\hline \multicolumn{4}{|l|}{ SLE manifestations } \\
\hline Mucocutaneous, $n(\%)$ & $41(27.3 \%)$ & $5(27.8 \%)$ & $>0.999$ \\
\hline Musculoskeletal, $n(\%)$ & $41(27.3 \%)$ & $3(16.7 \%)$ & 0.408 \\
\hline Neuropsychiatric, $n$ (\%) & $10(6.7 \%)$ & $3(16.7 \%)$ & 0.149 \\
\hline Serositis, $n(\%)$ & $23(15.3 \%)$ & $1(5.6 \%)$ & 0.475 \\
\hline Hematologic, $n(\%)$ & $67(44.7 \%)$ & $3(16.7 \%)$ & 0.023 \\
\hline \multicolumn{4}{|l|}{ Serology } \\
\hline Positive anti-Sm Ab, $n$ (\%) & $59(39.3 \%)$ & $9(50.0 \%)$ & 0.384 \\
\hline Positive anti-Ro Ab, n (\%) & $89(59.3 \%)$ & $11(61.1 \%)$ & 0.885 \\
\hline Positive anti-La Ab, n (\%) & $44(29.3 \%)$ & $3(16.7 \%)$ & 0.258 \\
\hline Positive anti-U1RNP Ab, n (\%) & $73(48.7 \%)$ & $14(77.8 \%)$ & 0.020 \\
\hline Positive anti-dsDNA Ab, $n$ (\%) & $132(88.0 \%)$ & $9(50.0 \%)$ & $<0.001$ \\
\hline Anti-dsDNA Ab level, median (IQR), IU/mL & $230.3(79.0-380.0)$ & $9.0(2.5-123.5)$ & $<0.001$ \\
\hline Positive anti-dsDNA Ab and negative anti-U1RNP Ab, $n(\%)$ & $66(44.0 \%)$ & $1(5.6 \%)$ & 0.002 \\
\hline Positive anti-U1RNP Ab and negative anti-dsDNA Ab, $n$ (\%) & $7(4.7 \%)$ & $6(33.3 \%)$ & 0.001 \\
\hline Positive anti-dsDNA Ab and anti-U1RNP Ab, $n$ (\%) & $66(44.0 \%)$ & $8(44.4 \%)$ & 0.971 \\
\hline Positive lupus anticoagulant, $n(\%)$ & $32(21.3 \%)$ & $3(16.7 \%)$ & 0.768 \\
\hline Positive anti- $\beta_{2}$ glycoprotein I Ab, $n$ (\%) & $18(12.0 \%)$ & $5(27.8 \%)$ & 0.077 \\
\hline Positive anti-cardiolipin Ab, n (\%) & $39(26.0 \%)$ & $3(16.7 \%)$ & 0.566 \\
\hline Low C3, n (\%) & $141(94.0 \%)$ & $11(61.1 \%)$ & $<0.001$ \\
\hline C3 level, median (IQR), mg/dL & $41.0(27.9-60.1)$ & $64.7(44.3-92.3)$ & 0.001 \\
\hline Low C4, $n(\%)$ & 119 (79.3\%) & $9(50.0 \%)$ & 0.015 \\
\hline C4 level, median (IQR), mg/dL & $5.5(2.5-11.9)$ & $12.8(7.3-23.5)$ & $<0.001$ \\
\hline Albumin level, mean ( $\pm \mathrm{SD}), \mathrm{g} / \mathrm{dL}$ & $2.8( \pm 0.7)$ & $2.9( \pm 0.9)$ & 0.800 \\
\hline Creatinine level, mean $( \pm \mathrm{SD}), \mathrm{mg} / \mathrm{dL}$ & $1.13( \pm 0.79)$ & $0.70( \pm 0.26)$ & $<0.001$ \\
\hline GFR, mean $( \pm \mathrm{SD}), \mathrm{mL} / \mathrm{min} / 1.73 \mathrm{~m}^{2}$ & $83.2( \pm 37.0)$ & $105.1( \pm 23.8)$ & 0.002 \\
\hline \multicolumn{4}{|l|}{ Urine } \\
\hline Urine PCR, median (IQR), mg/g & $3935.0(1903.4-6439.7)$ & $3464.5(2061.8-5775.0)$ & 0.778 \\
\hline Urine $\mathrm{RBC}$ of $\geq 5 / \mathrm{HPF}, n(\%)$ & 109 (72.7\%) & 7 (38.9\%) & 0.003 \\
\hline Urine $\mathrm{WBC}$ of $\geq 5 / \mathrm{HPF}, n(\%)$ & $86(57.3 \%)$ & $6(33.3 \%)$ & 0.053 \\
\hline Urine cast, $n(\%)$ & $31(20.7 \%)$ & $1(5.6 \%)$ & 0.202 \\
\hline SLEDAI-2K, mean ( \pm SD) & $17.1( \pm 5.8)$ & $12.2( \pm 5.8)$ & 0.001 \\
\hline
\end{tabular}

$L N$ lupus nephritis, SLE systemic lupus erythematosus, $A b$ antibody, anti-dsDNA anti-double-stranded DNA, GFR glomerular filtration rate, $P C R$ protein/creatinine ratio, $R B C$ red blood cell, HPF high power field, WBC white blood cell, SLEDAI-2K Systemic Lupus Erythematosus Disease Activity Index $2000, S D$ standard deviation, $I Q R$ interquartile range

In multivariable analysis model 1 , anti-U1RNP (positive/negative), anti-dsDNA Ab (positive/negative), C3 (low/not low), and C4 (low/not low) were analyzed as the categorical variables. An alternative multivariable analysis model (model 2) was also performed, in which anti-U1RNP, anti-dsDNA Ab, C3 level, and C4 level were analyzed as the continuous variables. Both the positivity (model 1) for and level (model 2) of anti-dsDNA Ab (model 1: adjusted $\mathrm{OR}=11.200,95 \% \mathrm{CI}=2.202-56.957$, $p=0.004$; model 2: adjusted $\mathrm{OR}=1.008,95 \% \mathrm{CI}=1.002$ $1.014, p=0.014)$ were associated with proliferative LN; conversely, both the positivity (model 1) for and level 
(model 2) of anti-U1RNP Ab (model 1: adjusted OR= $0.176,95 \% \mathrm{CI}=0.040-0.769, p=0.021$; model 2: adjusted $\mathrm{OR}=0.985,95 \% \mathrm{CI}=0.0976-0.994, p=0.002)$ were inversely associated with proliferative LN. Higher GFR (model 1: adjusted $\mathrm{OR}=0.973,95 \% \mathrm{CI}=0.951-0.994, p=$ 0.013 ; model 2: adjusted $\mathrm{OR}=0.969,95 \% \mathrm{CI}=0.946-$ $0.994, p=0.014)$ was also inversely associated with proliferative LN (Table 3).

\section{Ability of the covariates in discriminating proliferative $\mathrm{LN}$} The ROC curves for anti-U1RNP Ab, anti-dsDNA Ab, and the GFR are shown in Fig. 1. Anti-dsDNA Ab had the highest discrimination ability (AUC $=0.806,95 \%$ $\mathrm{CI}=0.695-0.916)$, followed by anti-U1RNP Ab (AUC = $0.677,95 \% \mathrm{CI}=0.527-0.827)$ and GFR $(\mathrm{AUC}=0.662$,
95\% $\mathrm{CI}=0.554-0.770)$. When a combination of antidsDNA Ab, anti-U1RNP Ab, and GFR was used as a composite parameter, the discrimination ability $(\mathrm{AUC}=$ $0.864,95 \% \mathrm{CI}=0.792-0.937)$ was higher than when each parameter was used as a single parameter (Fig. 1d).

\section{Sensitivity analysis}

When using the stricter proliferative LN definition (i.e., pure proliferative LN: class III and class IV), the ORs of anti-dsDNA Ab (model 1: adjusted $\mathrm{OR}=19.591,95 \%$ $\mathrm{CI}=2.518-152.431, p=0.004$; model 2: adjusted $\mathrm{OR}=$ $1.008,95 \% \mathrm{CI}=1.002-1.015, p=0.012)$, anti-U1RNP Ab (model 1: adjusted $\mathrm{OR}=0.178,95 \% \mathrm{CI}=0.034-0.922$, $p=0.040 ;$ model 2 : adjusted $\mathrm{OR}=0.987,95 \% \mathrm{CI}=$ $0.978-0.996, p=0.007$ ), and GFR (model 1: adjusted

Table 3 Factors associated with proliferative LN

\begin{tabular}{|c|c|c|c|c|c|c|}
\hline & \multicolumn{2}{|l|}{ Univariable analysis } & \multicolumn{2}{|c|}{ Multivariable analysis (model 1) } & \multicolumn{2}{|c|}{ Multivariable analysis (model 2) } \\
\hline & OR $(95 \% \mathrm{Cl})$ & $p$ & OR $(95 \% \mathrm{Cl})$ & $p$ & OR $(95 \% \mathrm{Cl})$ & $p$ \\
\hline Age & $0.982(0.951-1.014)$ & 0.269 & & & & \\
\hline Female sex & $1.437(0.295-7.006)$ & 0.653 & & & & \\
\hline Hypertension & $1.026(0.317-3.322)$ & 0.966 & & & & \\
\hline Diabetes mellitus & $\mathrm{N} / \mathrm{A}$ & 0.999 & & & & \\
\hline Mucocutaneous manifestations & $0.978(0.328-2.915)$ & 0.968 & & & & \\
\hline Musculoskeletal manifestations & $1.881(0.517-6.836)$ & 0.337 & & & & \\
\hline Neuropsychiatric manifestations & $0.357(0.088-1.442)$ & 0.148 & & & & \\
\hline Serositis & $3.079(0.390-24.280)$ & 0.286 & & & & \\
\hline Hematologic manifestations & $4.036(1.121-14.527)$ & 0.033 & $3.277(0.665-16.148)$ & 0.145 & $1.963(0.393-9.810)$ & 0.411 \\
\hline Positive anti-Sm Ab & $0.648(0.243-1.728)$ & 0.386 & & & & \\
\hline Positive anti-Ro Ab & $0.928(0.341-2.529)$ & 0.885 & & & & \\
\hline Positive anti-La Ab & $2.075(0.572-7.528)$ & 0.267 & & & & \\
\hline Positive anti-U1RNP $A b^{a}$ & $0.271(0.085-0.861)$ & 0.027 & $0.176(0.040-0.769)$ & 0.021 & $0.985(0.976-0.994)$ & 0.002 \\
\hline Positive anti-dsDNA $A b^{a}$ & $7.333(2.574-20.893)$ & $<0.001$ & $11.200(2.202-56.957)$ & 0.004 & $1.008(1.002-1.014)$ & 0.014 \\
\hline Positive lupus anticoagulant & $1.356(0.370-4.974)$ & 0.646 & & & & \\
\hline Positive anti- $\beta_{2}$ glycoprotein I Ab & $0.355(0.113-1.112)$ & 0.075 & & & & \\
\hline Positive anti-cardiolipin Ab & $1.757(0.483-6.396)$ & 0.393 & & & & \\
\hline Low C3a & $9.970(3.117-31.891)$ & $<0.001$ & $1.886(0.255-13.932)$ & 0.534 & $1.012(0.968-1.058)$ & 0.608 \\
\hline $\operatorname{Low} C 4^{\mathrm{a}}$ & $3.839(1.405-10.486)$ & 0.009 & $1.224(0.229-6.552)$ & 0.813 & $0.929(0.834-1.033)$ & 0.175 \\
\hline Albumin level & $0.886(0.434-1.805)$ & 0.738 & & & & \\
\hline Creatinine level & $10.645(1.350-83.953)$ & 0.025 & & & & \\
\hline GFR & $0.980(0.964-0.997)$ & 0.021 & $0.973(0.951-0.994)$ & 0.013 & $0.969(0.946-0.994)$ & 0.014 \\
\hline Urine PCR & $1.000(0.987-1.013)$ & 0.986 & & & & \\
\hline Urine $\mathrm{RBC}$ of $\geq 5 / \mathrm{HPF}$ & $4.178(1.516-11.509)$ & 0.006 & $2.053(0.426-9.893)$ & 0.370 & $1.721(0.307-9.652)$ & 0.537 \\
\hline Urine $\mathrm{WBC}$ of $\geq 5 / \mathrm{HPF}$ & $2.687(0.958-7.543)$ & 0.060 & & & & \\
\hline Urine cast & $4.429(0.567-34.578)$ & 0.156 & & & & \\
\hline SLEDAI-2K & $1.173(1.063-1.294)$ & 0.001 & $1.046(0.900-1.216)$ & 0.557 & $1.112(0.923-1.338)$ & 0.263 \\
\hline
\end{tabular}

$A b$ antibody, anti-dsDNA anti-double-stranded DNA, GFR glomerular filtration rate, $P C R$ protein/creatinine ratio, $R B C$ red blood cell, $H P F$ high power field, WBC white blood cell, SLEDAI-2K Systemic Lupus Erythematosus Disease Activity Index 2000, OR odds ratio, $C l$ confidence interval, $N / A$ not applicable ${ }^{a}$ Analyzed as binary variables (anti-U1RNP Ab, positive/negative; anti-dsDNA Ab, positive/negative; C3, low/not low; C4, low/not low) in univariable analysis and multivariable analysis (model 1) and analyzed as continuous variables in multivariable analysis (model 2) 

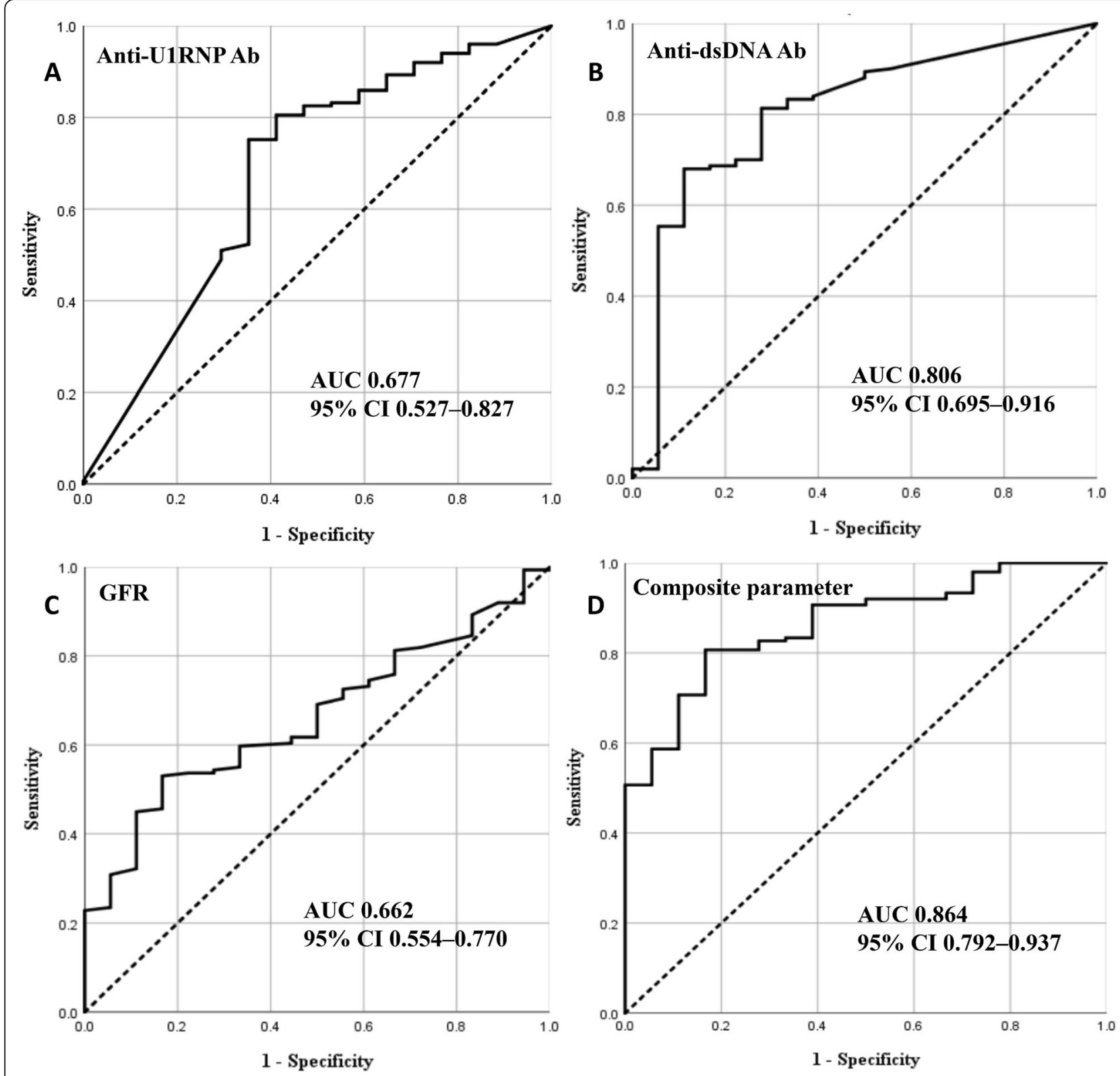

Fig. 1 Receiver-operating characteristic curves for the predictive value of a anti-U1RNP Ab, b anti-dsDNA Ab, c GFR, and $\mathbf{d}$ combination of antiU1RNP Ab, anti-dsDNA Ab, and GFR as a composite parameter, for predicting proliferative LN (class III, class IV, class III + V, and class IV + V). Ab, antibody; anti-dsDNA, anti-double-stranded DNA; GFR, glomerular filtration rate; LN, lupus nephritis; AUC, area under the curve; $\mathrm{Cl}$, confidence interval

OR $=0.966,95 \% \mathrm{CI}=0.941-0.992, p=0.010 ;$ model 2 : adjusted $\mathrm{OR}=0.967,95 \% \mathrm{CI}=0.942-0.992, p=0.011$ ) remained significant (Table 4). Further, the discrimination ability of anti-U1RNP Ab (AUC $=0.673,95 \%$ $\mathrm{CI}=0.522-0.823)$, anti-dsDNA Ab $(\mathrm{AUC}=0.822,95 \%$ $\mathrm{CI}=0.713-0.931)$, GFR $(\mathrm{AUC}=0.688,95 \% \mathrm{CI}=0.579-$ $0.798)$, and combination of anti-dsDNA Ab, antiU1RNP Ab, and GFR as a composite parameter (AUC $=0.873,95 \% \mathrm{CI}=0.801-0.945)$ was similar to the primary analysis (Fig. 2).
In another sensitivity analysis where mixed proliferative $\mathrm{LN}$ (class III $+\mathrm{V}$ and IV $+\mathrm{V}$ ) was compared with membranous LN (class V), positivity of anti-dsDNA Ab was still significantly associated with mixed proliferative LN (model 1: adjusted OR $=4.545$, 95\% CI 1.107-18.661, $p=0.036$ ) (Table 5), although the effect size was attenuated compared with the primary analysis (anti-dsDNA $\mathrm{Ab}$ in model 1 : adjusted $\mathrm{OR}=11.200$ ) (Table 3 ) and in the sensitivity analysis where pure proliferative $\mathrm{LN}$ was compared with membranous LN (anti-dsDNA Ab in 
Table 4 Sensitivity analysis: factors associated with pure proliferative LN

\begin{tabular}{|c|c|c|c|c|c|c|}
\hline & Univariable analysis & & Multivariable analysis (m & el 1) & Multivariable analysis & del 2) \\
\hline & $\overline{\mathrm{OR}}(95 \% \mathrm{Cl})$ & $p$ & $\overline{\mathrm{OR}}(95 \% \mathrm{Cl})$ & $p$ & $\overline{\mathrm{OR}}(95 \% \mathrm{Cl})$ & $p$ \\
\hline Age & $0.985(0.954-1.017)$ & 0.351 & & & & \\
\hline Female sex & $1.400(0.281-6.976)$ & 0.681 & & & & \\
\hline Hypertension & $1.043(0.318-3.422)$ & 0.945 & & & & \\
\hline Diabetes mellitus & N/A & 0.999 & & & & \\
\hline Mucocutaneous manifestations & $1.046(0.347-3.153)$ & 0.936 & & & & \\
\hline Musculoskeletal manifestations & $1.932(0.526-7.097)$ & 0.321 & & & & \\
\hline Neuropsychiatric manifestations & $0.304(0.071-1.305)$ & 0.109 & & & & \\
\hline Serositis & $3.535(0.446-28.035)$ & 0.232 & & & & \\
\hline Hematologic manifestations & $4.531(1.248-16.453)$ & 0.022 & $4.997(0.819-30.479)$ & 0.081 & $2.150(0.408-11.333)$ & 0.367 \\
\hline Positive anti-Sm Ab & $0.718(0.267-1.936)$ & 0.513 & & & & \\
\hline Positive anti-Ro Ab & $1.051(0.381-2.904)$ & 0.923 & & & & \\
\hline Positive anti-La Ab & $2.262(0.618-8.279)$ & 0.218 & & & & \\
\hline Positive anti-U1RNP $A b^{a}$ & $0.268(0.083-0.859)$ & 0.027 & $0.178(0.034-0.922)$ & 0.040 & $0.987(0.978-0.996)$ & 0.007 \\
\hline Positive anti-dsDNA $A b^{a}$ & $8.385(2.824-24.896)$ & $<0.001$ & $19.591(2.518-152.431)$ & 0.004 & $1.008(1.002-1.015)$ & 0.012 \\
\hline Positive lupus anticoagulant & $1.224(0.328-4.572)$ & 0.763 & & & & \\
\hline Positive anti- $\beta_{2}$ glycoprotein I Ab & $0.337(0.104-1.088)$ & 0.069 & & & & \\
\hline Positive anti-cardiolipin Ab & $1.854(0.504-6.819)$ & 0.353 & & & & \\
\hline Low $\mathrm{C3}^{\mathrm{a}}$ & $14.891(4.044-54.831)$ & $<0.001$ & $2.216(0.216-22.738)$ & 0.503 & $1.004(0.959-1.052)$ & 0.866 \\
\hline Low $C 4^{a}$ & $5.421(1.906-15.422)$ & 0.002 & $1.763(0.313-9.916)$ & 0.520 & $0.947(0.839-1.069)$ & 0.376 \\
\hline Albumin level & $0.898(0.420-1.918)$ & 0.781 & & & & \\
\hline Creatinine level & $11.857(1.524-92.273)$ & 0.018 & & & & \\
\hline GFR & $0.978(0.961-0.995)$ & 0.012 & $0.966(0.941-0.992)$ & 0.010 & $0.967(0.942-0.992)$ & 0.011 \\
\hline Urine PCR & $0.999(0.986-1.013)$ & 0.934 & & & & \\
\hline Urine $\mathrm{RBC}$ of $\geq 5 / \mathrm{HPF}$ & $4.238(1.515-11.852)$ & 0.006 & $1.803(0.358-9.087)$ & 0.475 & 1.724 (0.299-9.952) & 0.542 \\
\hline Urine $\mathrm{WBC}$ of $\geq 5 / \mathrm{HPF}$ & $2.604(0.917-7.391)$ & 0.072 & & & & \\
\hline Urine cast & $4.381(0.556-34.519)$ & 0.161 & & & & \\
\hline SLEDAI-2K & $1.181(1.067-1.308)$ & 0.001 & $1.026(0.884-1.190)$ & 0.739 & $1.094(0.899-1.330)$ & 0.370 \\
\hline
\end{tabular}

$A b$ antibody, anti-dsDNA anti-double-stranded DNA, GFR glomerular filtration rate, $P C R$ protein/creatinine ratio, RBC red blood cell, HPF high power field, WBC white blood cell, SLEDAl-2K Systemic Lupus Erythematosus Disease Activity Index 2000, OR odds ratio, Cl confidence interval, N/A, not applicable

${ }^{a}$ Analyzed as binary variables (anti-U1RNP Ab, positive/negative; anti-dsDNA Ab, positive/negative; C3, low/not low; C4, low/not low) in univariable analysis and multivariable analysis (model 1) and analyzed as continuous variables in multivariable analysis (model 2)

model 1: adjusted OR $=19.591)$ (Table 4). The effect size of anti-U1RNP $\mathrm{Ab}$ (unadjusted $\mathrm{OR}=0.286,95 \% \mathrm{CI}$ 0.075-1.086, $p=0.066$ ) and GFR (unadjusted $\mathrm{OR}=$ $0.988,95 \%$ CI $0.968-1.009, p=0.273$ ) was also attenuated and failed to reach statistical significance (Table 5).

In comparison between pure proliferative LN (class III and class IV) and non-proliferative LN (class I, class II, class $\mathrm{V}$, and class VI), anti-dsDNA Ab (model 1: adjusted OR = 13.741, 95\% CI = 3.058-61.753, $p=0.001$; model 2: adjusted $\mathrm{OR}=1.008,95 \% \mathrm{CI}=1.003-1.012, p=0.002)$ was associated with pure proliferative $\mathrm{LN}$, and anti-U1RNP $\mathrm{Ab}$ (model 1: adjusted OR $=0.273,95 \% \mathrm{CI}=0.085-0.873, p=$ 0.029; model 2: adjusted $\mathrm{OR}=0.991,95 \% \mathrm{CI}=0.984-0.998$, $p=0.012$ ) and GFR (model 1: adjusted OR $=0.970,95 \%$ $\mathrm{CI}=0.952-0.989, p=0.003$; model 2 : adjusted $\mathrm{OR}=0.972$,
95\% CI $=0.954-0.990, \mathrm{p}=0.002$ ) were inversely associated with pure proliferative $\mathrm{LN}$, supporting their value in predicting proliferative LN (Table 6).

\section{Discussion}

In this retrospective cohort study, we showed that the positivity for and level of anti-dsDNA Ab were significantly associated with proliferative LN and that the positivity for and level of anti-U1RNP Ab and the GFR were inversely associated with proliferative LN. Among these covariates, anti-dsDNA Ab had the highest ability to discriminate proliferative $\mathrm{LN}$ from membranous LN. These findings are meaningful in that they may aid in therapeutic decision-making for clinicians when renal biopsies are difficult to perform. 

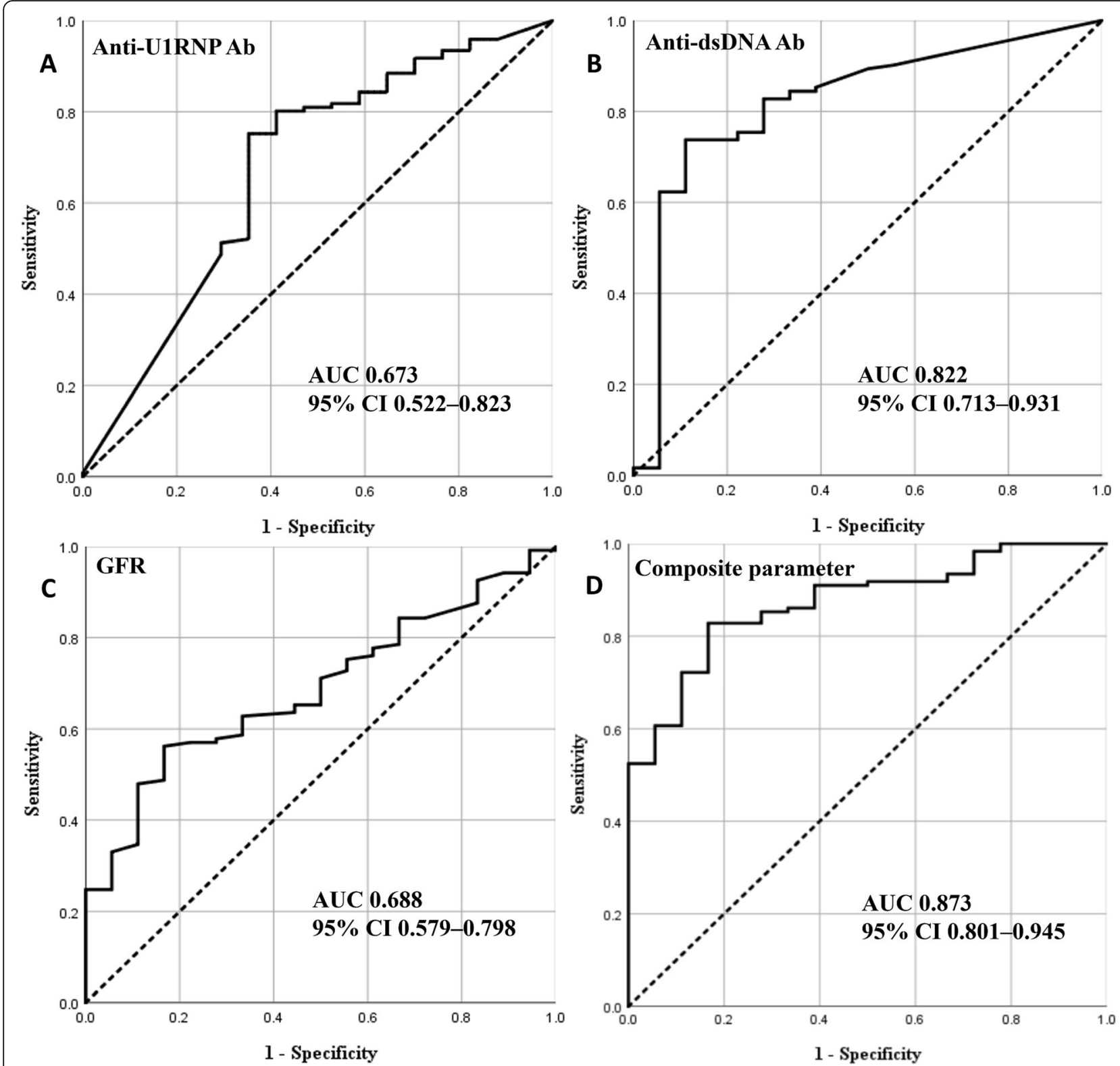

Fig. 2 Receiver-operating characteristic curves for the predictive value of a anti-U1RNP Ab, b anti-dsDNA Ab, c GFR, and $\mathbf{d}$ combination of antiU1RNP Ab, anti-dsDNA Ab, and GFR as a composite parameter, for predicting pure proliferative LN (class III and class IV). Ab, antibody; antidsDNA, anti-double-stranded DNA; GFR, glomerular filtration rate; LN, lupus nephritis; AUC, area under the curve; Cl, confidence interval

Several previous studies have reported non-histologic factors associated with LN in patients with SLE [11-14]. Although there are some inconsistencies among these reports, anti-dsDNA Ab is consistently reported as an autoantibody that is associated with the occurrence of renal disease in SLE [11-14]. Mechanistically, anti-dsDNA Ab is involved in the development of LN by binding to glomerular and tubulointerstitial cells, inducing cell proliferation, inflammation, apoptosis, and fibrosis [15]. Similar to our present finding, a previous study also reported antidsDNA $\mathrm{Ab}$ as an important factor associated with proliferative LN compared with non-proliferative LN [16]. We further advanced the previous report by performing ROC analysis and providing the predictive value of antidsDNA Ab. Moreover, we also included anti-ENA Abs such as anti-Ro Ab, anti-La Ab, and anti-U1RNP Ab as variables in our analysis and found that anti-U1RNP $A b$ was inversely associated with proliferative $\mathrm{LN}$, which was not reported in the previous study.

Anti-U1RNP Ab, which is by definition found in 100\% of patients with mixed connective tissue disease, is found in $20-40 \%$ of patients with SLE [17]. Previous studies 
Table 5 Sensitivity analysis: factors associated with mixed proliferative LN (classes III $+V$ and IV $+V, n=28$ ) vs membranous LN (class $\vee, n=18$ )

\begin{tabular}{|c|c|c|c|c|c|c|}
\hline & \multicolumn{2}{|l|}{ Univariable analysis } & \multicolumn{2}{|c|}{ Multivariable analysis (model 1) } & \multicolumn{2}{|c|}{ Multivariable analysis (model 2) } \\
\hline & $\mathrm{OR}(95 \% \mathrm{Cl})$ & $p$ & OR $(95 \% \mathrm{Cl})$ & $p$ & OR $(95 \% \mathrm{Cl})$ & $p$ \\
\hline Age & $0.965(0.920-1.012)$ & 0.142 & & & & \\
\hline Female sex & $1.625(0.208-12.705)$ & 0.644 & & & & \\
\hline Hypertension & $0.955(0.228-3.995)$ & 0.949 & & & & \\
\hline Diabetes mellitus & N/A & $>0.999$ & & & & \\
\hline Mucocutaneous manifestations & $0.709(0.180-2.792)$ & 0.623 & & & & \\
\hline Musculoskeletal manifestations & $1.667(0.370-7.515)$ & 0.506 & & & & \\
\hline Neuropsychiatric manifestations & $0.600(0.107-3.363)$ & 0.561 & & & & \\
\hline Serositis & $1.308(0.110-15.570)$ & 0.832 & & & & \\
\hline Hematologic manifestations & $2.368(0.544-10.317)$ & 0.251 & & & & \\
\hline Positive anti-Sm Ab & $0.400(0.116-1.376)$ & 0.146 & & & & \\
\hline Positive anti-Ro Ab & $0.552(0.165-1.838)$ & 0.333 & & & & \\
\hline Positive anti-La Ab & $1.364(0.294-6.319)$ & 0.692 & & & & \\
\hline Positive anti-U1RNP Ab ${ }^{a}$ & $0.286(0.075-1.086)$ & 0.066 & & & & \\
\hline Positive anti-dsDNA $A b^{b}$ & $4.600(1.207-17.524)$ & 0.025 & $4.545(1.107-18.661)$ & 0.036 & $1.003(0.999-1.007)$ & 0.159 \\
\hline Positive lupus anticoagulant & $2.000(0.452-8.841)$ & 0.361 & & & & \\
\hline Positive anti- $\beta_{2}$ glycoprotein I Ab & $0.433(0.099-1.900)$ & 0.267 & & & & \\
\hline Positive anti-cardiolipin Ab & $1.364(0.294-6.319)$ & 0.692 & & & & \\
\hline Low $C 3^{a}$ & $3.818(0.922-15.808)$ & 0.065 & & & & \\
\hline $\operatorname{Low} C 4^{\mathrm{a}}$ & $1.333(0.406-4.379)$ & 0.635 & & & & \\
\hline Albumin level & $0.866(0.435-1.721)$ & 0.680 & & & & \\
\hline Creatinine level & $5.076(0.446-57.756)$ & 0.190 & & & & \\
\hline GFR & $0.988(0.968-1.009)$ & 0.273 & & & & \\
\hline Urine PCR & $1.002(0.985-1.020)$ & 0.808 & & & & \\
\hline Urine $\mathrm{RBC}$ of $\geq 5 / \mathrm{HPF}$ & $3.929(1.122-13.755)$ & 0.032 & $3.326(0.559-19.795)$ & 0.187 & $2.402(0.464-12.428)$ & 0.296 \\
\hline Urine WBC of $\geq 5 / \mathrm{HPF}$ & $3.091(0.895-10.672)$ & 0.074 & & & & \\
\hline Urine cast & $4.636(0.509-42.246)$ & 0.174 & & & & \\
\hline SLEDAI-2K & $1.134(1.015-1.266)$ & 0.026 & $1.020(0.872-1.195)$ & 0.801 & $1.074(0.935-1.233)$ & 0.315 \\
\hline
\end{tabular}

$A b$ antibody, anti-dsDNA anti-double-stranded DNA, GFR glomerular filtration rate, $P C R$ protein/creatinine ratio, $R B C$ red blood cell, $H P F$ high power field, WBC white blood cell, SLEDAI-2K Systemic Lupus Erythematosus Disease Activity Index 2000, OR odds ratio, $\mathrm{Cl}$ confidence interval, $\mathrm{N} / \mathrm{A}$ not applicable

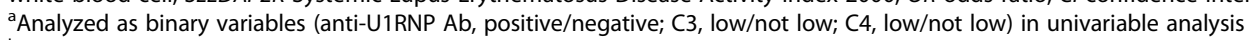

${ }^{b}$ Analyzed as binary variables (Anti-dsDNA Ab, positive/negative) in univariable analysis and multivariable analysis (model 1 ) and analyzed as continuous variables in multivariable analysis (model 2)

have reported an association between anti-U1RNP $\mathrm{Ab}$ and occurrence of pulmonary hypertension in patients with SLE [18-20]. There are conflicting data regarding the association between anti-U1RNP Ab and renal disease in SLE $[11-13,21]$. One study reported a $66 \%$ reduced risk of LN development in patients with SLE with positive anti-U1RNP Ab findings [21], whereas other studies reported no association between the presence of anti-U1RNP Ab and LN development [11, 12] or even a higher risk of LN development [13]. Although the association between anti-U1RNP $\mathrm{Ab}$ and the presence of renal disease in SLE is controversial, the presence of anti-U1RNP Ab may have clinical significance when confined to patients with LN in that it is inversely associated with proliferative LN. In other words, the presence of anti-U1RNP Ab in patients with $\mathrm{LN}$ may suggest that the glomerular pathology might be membranous $\mathrm{LN}$. This finding is meaningful because it is the first to indicate an association between anti-U1RNP Ab and the renal pathologic class.

We also found that the GFR was inversely associated with proliferative LN; a lower GFR was suggestive of proliferative LN. The other covariates associated with renal manifestations, such as amount of proteinuria and presence of hematuria, pyuria, and urine casts, were not associated with proliferative LN. Although these covariates are 
Table 6 Sensitivity analysis: factors associated with pure proliferative LN (classes III and IV, $n=122)$ vs non-proliferative LN (classes I, II, $\mathrm{V}$ and $\mathrm{VI}, n=26)$

\begin{tabular}{|c|c|c|c|c|c|c|}
\hline & \multicolumn{2}{|l|}{ Univariable analysis } & \multicolumn{2}{|c|}{ Multivariable analysis (model 1) } & \multicolumn{2}{|c|}{ Multivariable analysis (model 2) } \\
\hline & OR $(95 \% \mathrm{Cl})$ & $p$ & OR $(95 \% \mathrm{Cl})$ & $p$ & OR $(95 \% \mathrm{Cl})$ & $p$ \\
\hline Age & $0.977(0.951-1.002)$ & 0.074 & & & & \\
\hline Female sex & $2.036(0.586-7.082)$ & 0.263 & & & & \\
\hline Hypertension & $0.809(0.308-2.120)$ & 0.666 & & & & \\
\hline Diabetes mellitus & N/A & 0.999 & & & & \\
\hline Mucocutaneous manifestations & $1.690(0.591-4.834)$ & 0.328 & & & & \\
\hline Musculoskeletal manifestations & $1.623(0.566-4.649)$ & 0.367 & & & & \\
\hline Neuropsychiatric manifestations & $0.335(0.090-1.241)$ & 0.102 & & & & \\
\hline Serositis & $2.495(0.547-11.375)$ & 0.238 & & & & \\
\hline Hematologic manifestations & $2.460(0.964-6.276)$ & 0.060 & & & & \\
\hline Positive anti-Sm Ab & $0.838(0.358-1.962)$ & 0.684 & & & & \\
\hline Positive anti-Ro Ab & $1.212(0.513-2.863)$ & 0.662 & & & & \\
\hline Positive anti-La Ab & $2.488(0.802-7.719)$ & 0.115 & & & & \\
\hline Positive anti-U1RNP $A b^{a}$ & $0.345(0.135-0.880)$ & 0.026 & $0.273(0.085-0.873)$ & 0.029 & $0.991(0.984-0.998)$ & 0.012 \\
\hline Positive anti-dsDNA $A b^{a}$ & $8.385(3.210-21.900)$ & $<0.001$ & $13.741(3.058-61.753)$ & 0.001 & $1.008(1.003-1.012)$ & 0.002 \\
\hline Positive lupus anticoagulant & $1.347(0.424-4.276)$ & 0.613 & & & & \\
\hline Positive anti- $\beta_{2}$ glycoprotein I Ab & $0.544(0.177-1.674)$ & 0.289 & & & & \\
\hline Positive anti-cardiolipin Ab & $2.039(0.654-6.362)$ & 0.220 & & & & \\
\hline Low $C 3^{a}$ & $10.400(3.062-35.318)$ & $<0.001$ & $2.072(0.281-15.292)$ & 0.475 & $0.994(0.958-1.031)$ & 0.747 \\
\hline Low $C 4^{\mathrm{a}}$ & $3.975(1.586-9.967)$ & 0.003 & $1.811(0.430-7.635)$ & 0.418 & $0.958(0.868-1.058)$ & 0.395 \\
\hline Albumin level & $0.665(0.347-1.274)$ & 0.218 & & & & \\
\hline Creatinine level & $2.722(0.970-7.634)$ & 0.057 & & & & \\
\hline GFR & $0.983(0.969-0.996)$ & 0.013 & $0.970(0.952-0.989)$ & 0.003 & $0.972(0.954-0.990)$ & 0.002 \\
\hline Urine PCR & $1.003(0.990-1.015)$ & 0.669 & & & & \\
\hline Urine $\mathrm{RBC}$ of $\geq 5 / \mathrm{HPF}$ & $3.678(1.534-8.819)$ & 0.004 & $2.038(0.538-7.720)$ & 0.295 & $2.400(0.588-9.796)$ & 0.222 \\
\hline Urine $\mathrm{WBC}$ of $\geq 5 / \mathrm{HPF}$ & $2.083(0.875-4.959)$ & 0.097 & & & & \\
\hline Urine cast & $1.976(0.549-7.113)$ & 0.297 & & & & \\
\hline SLEDAI-2K & $1.149(1.056-1.251)$ & 0.001 & $1.012(0.896-1.144)$ & 0.845 & $1.017(0.883-1.171)$ & 0.818 \\
\hline
\end{tabular}

$A b$ antibody, anti-dsDNA anti-double-stranded DNA, GFR glomerular filtration rate, $P C R$ protein/creatinine ratio, $R B C$ red blood cell, $H P F$ high power field, WBC white blood cell, SLEDAI-2K Systemic Lupus Erythematosus Disease Activity Index 2000, OR odds ratio, $\mathrm{Cl}$ confidence interval, $\mathrm{N} / \mathrm{A}$ not applicable

${ }^{a}$ Analyzed as binary variables (anti-U1RNP Ab, positive/negative; anti-dsDNA Ab, positive/negative; C3, low/not low; C4, low/not low) in univariable analysis and multivariable analysis (model 1 ) and analyzed as continuous variables in multivariable analysis (model 2)

the components used in measuring disease activity (SLEDAI-2 K), they are not necessarily associated with a particular renal histology. Rather, the GFR was closely associated with the histologic classes of LN, although it is not a component of the SLEDAI-2 K.

Sensitivity analyses showed that effect sizes (i.e., ORs) of anti-dsDNA Ab, anti-U1RNP Ab, and GFR were greatest when pure proliferative LN was compared with membranous LN, followed by when proliferative LN (both pure and mixed proliferative LN) was compared with membranous $\mathrm{LN}$, and when mixed proliferative $\mathrm{LN}$ was compared with membranous LN. This suggests that anti-dsDNA Ab, anti-U1RNP Ab, and GFR are particularly useful in detecting pure proliferative LN. Further, in the analysis where class I, class II, and class VI were included, anti-dsDNA $\mathrm{Ab}$, anti-U1RNP Ab, and GFR were still significantly associated with pure proliferative LN, showing the robustness of the findings of the primary analysis.

We used the ROC analysis to assess the ability of antiU1RNP Ab, anti-dsDNA Ab, and the GFR to predict proliferative LN. Given that the AUCs can be interpreted as follows: $1.00-0.90=$ excellent, $0.80-0.90=$ good, $0.70-$ $0.80=$ fair, $0.60-0.70=$ poor, and $0.50-0.60=$ fail [22], anti-dsDNA Ab (AUC $=0.806$ ) had good accuracy, while anti-U1RNP Ab (AUC=0.677) and the GFR (AUC= 0.662 ) had poor accuracy in discriminating between proliferative LN and membranous LN in the present study. When anti-dsDNA Ab was combined with anti-U1RNP 
$\mathrm{Ab}$ and GFR, the accuracy was numerically higher (AUC $=0.864$ ) than when used as a single parameter. Similar results were also found in our sensitivity analysis. None of the covariates had an AUC of 1.00; therefore, these covariates cannot completely replace renal biopsy findings in discriminating between proliferative $\mathrm{LN}$ and membranous LN. However, in circumstances where renal biopsy is difficult to perform, anti-dsDNA $\mathrm{Ab}$ or its combination with anti-U1RNP Ab and GFR may be useful in discriminating between them, considering its good discriminating accuracy.

The present study has some limitations. First, as renal biopsies were performed only in the patients with overt clinical renal manifestations, patients with "silent" LN, which is defined as histologic LN in the absence of clinical renal manifestations [23, 24], were not included in our study. Therefore, our findings may not be generalized to patients with silent LN. However, considering that the value of renal biopsy and need for treatment is uncertain in silent LN [23], the covariates associated with proliferative $\mathrm{LN}$ in the patients with overt renal manifestations that we identified in this study still have clinical significance. Second, although we identified the covariates associated with proliferative LN, explanation for their associations cannot be drawn from the present study data. Further studies elucidating the mechanisms underlying these associations would be helpful. Third, the course of the study was long, and due to the retrospective nature of our study, we were unable to clarify whether the assay for measuring anti-dsDNA Ab has changed over time. Although the unit of anti-dsDNA Ab was the same $(\mathrm{IU} / \mathrm{ml})$ in all patients included, the potential of confounding remains by the possibility that the assay may have changed during the study period.

\section{Conclusion}

In conclusion, we found that anti-U1RNP Ab, antidsDNA Ab, and the GFR are associated with glomerular pathology in patients with LN. Among these covariates, the anti-dsDNA Ab had a good accuracy in discriminating proliferative LN from membranous LN. Although anti-dsDNA Ab cannot replace the performance of renal biopsy findings, it can be helpful in patients with SLE with clinically overt renal manifestations who cannot undergo renal biopsies.

\footnotetext{
Abbreviations

LN: Lupus nephritis; SLE: Systemic lupus erythematosus; ESRD: End-stage renal disease; ACR: American College of Rheumatology; PCR: Protein/ creatinine ratio; RBC: Red blood cell; HPF: High power field; Ab: Antibody; anti-dsDNA: Anti-double-stranded DNA; GFR: Glomerular filtration rate; SLEDAI-2 K: Systemic lupus erythematosus disease activity index 2000; ROC: Receiver-operating characteristic; AUC: Area under the curve; OR: Odds ratio; Cl: Confidence interval
}

\section{Acknowledgements}

None.

\section{Authors' contributions}

OCK contributed to the acquisition of data, data analyses, data interpretation, and manuscript preparation. JHP contributed to the acquisition of data, data interpretation, and manuscript preparation. $\mathrm{H}-\mathrm{CP}$ contributed to the data analyses, data interpretation, and manuscript preparation. SMJ contributed to acquisition of data, data interpretation, and manuscript preparation. S-WL contributed to acquisition of data, data interpretation, and manuscript preparation. JJS contributed to the acquisition of data, data interpretation, and manuscript preparation. Y-BP contributed to the data analyses, data interpretation, and manuscript preparation. M-CP contributed to the study concept and design, acquisition of data, data interpretation, and manuscript preparation. All authors read and approved the final manuscript.

\section{Funding}

This study was supported by a faculty research grant of Yonsei University College of Medicine (6-2019-0111).

\section{Availability of data and materials}

All data generated or analyzed during this study are included in this article.

\section{Ethics approval and consent to participate}

This study was approved by the Institutional Review Board (IRB) of Gangnam Severance Hospital (IRB No: 3-2019-0072). Owing to the retrospective nature of this study, the requirement for informed consent was waived.

\section{Consent for publication}

Not applicable.

\section{Competing interests}

None.

\section{Author details}

${ }^{1}$ Division of Rheumatology, Department of Internal Medicine, Yonsei University College of Medicine, Seoul, South Korea. ${ }^{2}$ Division of Nephrology, Department of Internal Medicine, Yonsei University College of Medicine, Seoul, South Korea.

Received: 6 November 2019 Accepted: 21 May 2020

Published online: 09 June 2020

\section{References}

1. Bernatsky S, Boivin JF, Joseph L, Manzi S, Ginzler E, Gladman DD, et al. Mortality in systemic lupus erythematosus. Arthritis Rheum. 2006;54:2550-7. https://doi.org/10.1002/art.21955.

2. Weening JJ, D'Agati VD, Schwartz MM, Seshan SV, Alpers CE, Appel GB, et al. The classification of glomerulonephritis in systemic lupus erythematosus revisited. J Am Soc Nephrol. 2004;15:241-50. https://doi.org/10.1097/01.asn. 0000108969.21691.5d.

3. Davidson A. What is damaging the kidney in lupus nephritis? Nat Rev Rheumatol. 2016;12:143-53. https://doi.org/10.1038/nrrheum.2015.159.

4. Almaani S, Meara A, Rovin BH. Update on lupus nephritis. Clin J Am Soc Nephrol. 2017;12:825-35. https://doi.org/10.2215/cjn.05780616.

5. Yu F, Haas M, Glassock R, Zhao MH. Redefining lupus nephritis: clinical implications of pathophysiologic subtypes. Nat Rev Nephrol. 2017;13:48395. https://doi.org/10.1038/nrneph.2017.85.

6. Houssiau FA, Lauwerys BR. Current management of lupus nephritis. Best Pract Res Clin Rheumatol. 2013;27:319-28. https://doi.org/10.1016/j.berh. 2013.07.004.

7. Hahn BH, McMahon MA, Wilkinson A, Wallace WD, Daikh DI, Fitzgerald JD, et al. American College of Rheumatology guidelines for screening, treatment, and management of lupus nephritis. Arthritis Care Res (Hoboken). 2012;64:797-808. https://doi.org/10.1002/acr.21664.

8. Hochberg MC. Updating the American College of Rheumatology revised criteria for the classification of systemic lupus erythematosus. Arthritis Rheum. 1997;40:1725. https://doi.org/10.1002/art.1780400928.

9. Bertsias GK, Tektonidou M, Amoura Z, Aringer M, Bajema I, Berden JH, et al. Joint European League Against Rheumatism and European Renal Association-European Dialysis and Transplant Association (EULAR/ERA-EDTA) recommendations for the management of adult and paediatric lupus nephritis. Ann Rheum Dis. 2012;71:1771-82. https://doi.org/10.1136/ annrheumdis-2012-201940. 
10. Gladman DD, Ibanez D, Urowitz MB. Systemic lupus erythematosus disease activity index 2000. J Rheumatol. 2002;29:288-91.

11. Alba P, Bento L, Cuadrado MJ, Karim Y, Tungekar MF, Abbs I, et al. AntidsDNA, anti-Sm antibodies, and the lupus anticoagulant: significant factors associated with lupus nephritis. Ann Rheum Dis. 2003;62:556-60. https://doi. org/10.1136/ard.62.6.556.

12. Kwon OC, Lee JS, Ghang B, Kim YG, Lee CK, Yoo B, et al. Predicting eventual development of lupus nephritis at the time of diagnosis of systemic lupus erythematosus. Semin Arthritis Rheum. 2018;48:462-6. https://doi.org/10. 1016/j.semarthrit.2018.02.012.

13. Bastian HM, Roseman JM, McGwin G Jr, Alarcon GS, Friedman AW, Fessler BJ, et al. Systemic lupus erythematosus in three ethnic groups. XII. Risk factors for lupus nephritis after diagnosis. Lupus. 2002;11:152-60. https://doi. org/10.1191/0961203302lu158oa.

14. Sule SD, Moodalbail DG, Burnham J, Fivush B, Furth SL. Predictors of kidney disease in a cohort of pediatric patients with lupus. Lupus. 2015;24:862-8. https://doi.org/10.1177/0961203315570162.

15. Yung S, Chan TM. Mechanisms of kidney injury in lupus nephritis - the role of anti-dsDNA antibodies. Front Immunol. 2015;6:475. https://doi.org/10. 3389/fimmu.2015.00475.

16. Moroni G, Quaglini S, Radice A, Trezzi B, Raffiotta F, Messa P, et al. The value of a panel of autoantibodies for predicting the activity of lupus nephritis at time of renal biopsy. J Immunol Res. 2015;2015:106904. https://doi.org/10. 1155/2015/106904

17. Cappelli S, Bellando Randone S, Martinovic D, Tamas MM, Pasalic K, Allanore $Y$, et al. "To be or not to be," ten years after: evidence for mixed connective tissue disease as a distinct entity. Semin Arthritis Rheum. 2012;41:589-98. https://doi.org/10.1016/.jsemarthrit.2011.07.010.

18. Chow SL, Chandran V, Fazelzad R, Johnson SR. Prognostic factors for survival in systemic lupus erythematosus associated pulmonary hypertension. Lupus. 2012;21:353-64. https://doi.org/10.1177/0961203311429815.

19. Lian F, Chen D, Wang Y, Ye Y, Wang X, Zhan Z, et al. Clinical features and independent predictors of pulmonary arterial hypertension in systemic lupus erythematosus. Rheumatol Int. 2012;32:1727-31. https://doi.org/10. 1007/s00296-011-1880-4.

20. Artim-Esen B, Cene E, Sahinkaya Y, Ertan S, Pehlivan O, Kamali S, et al. Cluster analysis of autoantibodies in 852 patients with systemic lupus erythematosus from a single center. J Rheumatol. 2014;41:1304-10. https:// doi.org/10.3899/jrheum.130984

21. Tapanes FJ, Vasquez M, Ramirez R, Matheus C, Rodriguez MA, Bianco N. Cluster analysis of antinuclear autoantibodies in the prognosis of SLE nephropathy: are anti-extractable nuclear antibodies protective? Lupus. 2000;9:437-44. https://doi.org/10.1191/096120300678828604.

22. Safari S, Baratloo A, Elfil M, Negida A. Evidence based emergency medicine; part 5 receiver operating curve and area under the curve. Emerg (Tehran). 2016:4:111-3.

23. Gonzalez-Crespo MR, Lopez-Fernandez J, Usera G, Poveda MJ, Gomez-Reino JJ. Outcome of silent lupus nephritis. Semin Arthritis Rheum. 1996;26:46876.

24. Bennett WM, Bardana EJ, Norman DJ, Houghton DC. Natural history of "silent" lupus nephritis. Am J Kidney Dis. 1982;1:359-63.

\section{Publisher's Note}

Springer Nature remains neutral with regard to jurisdictional claims in published maps and institutional affiliations.

Ready to submit your research? Choose BMC and benefit from:

- fast, convenient online submission

- thorough peer review by experienced researchers in your field

- rapid publication on acceptance

- support for research data, including large and complex data types

- gold Open Access which fosters wider collaboration and increased citations

- maximum visibility for your research: over $100 \mathrm{M}$ website views per year

At BMC, research is always in progress.

Learn more biomedcentral.com/submissions 